\title{
Are there mappable genes for family resemblance for the magnitude of intra-individual variation in systolic blood pressure? Jennifer Lin*1, Anthony Hinrichs ${ }^{2}$ and Brian K Suarez ${ }^{2,3}$
}

\author{
Address: ${ }^{1}$ Division of Preventive Medicine, Brigham and Women's Hospital, Harvard Medical School, Boston, Massachusetts, USA, ${ }^{2}$ Department \\ of Psychiatry, Washington University School of Medicine, St. Louis, Missouri, USA and ${ }^{3}$ Department of Genetics, Washington University School \\ of Medicine, St. Louis, Missouri, USA \\ Email: Jennifer Lin* - jhlin@rics.bwh.harvard.edu; Anthony Hinrichs - tony@silver.wustl.edu; Brian K Suarez - bks@themfs.wustl.edu \\ * Corresponding author
}

from Genetic Analysis Workshop I3: Analysis of Longitudinal Family Data for Complex Diseases and Related Risk Factors

New Orleans Marriott Hotel, New Orleans, LA, USA, November I I-14, 2002

Published: 3I December 2003

BMC Genetics 2003, 4(Suppl I):SI I

This article is available from: http://www.biomedcentral.com/|47|-2I56/4/s I/SI I

\begin{abstract}
Background: The genetic regulation of variation in intra-individual fluctuations in systolic blood pressure over time is poorly understood. Analysis of the magnitude of the average fluctuation of a person's systolic blood pressure around his or her age-adjusted trend line, however, shows moderate, albeit significant, family resemblance in Cohort I of the Framingham Heart Study. To determine whether genomic regions affecting this phenotype could be identified, we pursued a "model-free" multipoint quantitative linkage analysis.

Results: Two different linkage methods revealed multiple nominally significant signals, two to four of which are "replicated" in Cohort 2. When both cohorts are assembled into extended pedigrees, three linkage signals remain nominally significant by one or both methods.
\end{abstract}

Conclusion: Any or all of the genomic regions in the vicinity of D5S I 456, DI IS2359, and D20S470 may contain elements that regulate systolic blood pressure homeostasis.

\section{Background}

We used the rich longitudinal Framingham Heart Study data to explore the hypothesis that there is a heritable component to intra-individual variation in systolic blood pressure (SBP). Our strategy was to: 1) document family resemblance for the variability of SBP in Cohort $1 ; 2$ ) use multipoint quantitative linkage analysis to identify genomic regions that may harbor genes that affect withinperson variability in SBP; and 3) replicate any findings in Cohort 2. Despite the inherent differences between the two cohorts, if one or more linkage signals are replicated, we will combine the cohorts and repeat the analysis on the extended pedigrees.
We are particularly interested in assessing variation in the context of homeostatic regulation. It has been shown that a locus influencing age-adjusted SBP maps to chromosome $17 \mathrm{q}$ in these data [1]. We hypothesize that there is, additionally, familial resemblance for the magnitude of the fluctuations about each individual's age-related mean SBP. Since we anticipate that different individuals may have unique age-related trends (e.g., increasing SBP with age in some individuals, declining SBP in other individuals) and since we are not interested in the age-related means, per se, but rather the size of the fluctuations, we defined the phenotype as the average of absolute residual (AVGRES) obtained by regressing each person's SBP measurements on his or her age when the measurements were obtained. Because we wanted to be assured of having a 
sufficient number of measurements per person, we restricted our Cohort 1 analysis to subjects whose SBP was measured on a minimum of ten occasions. The mean number of SBP measurements for the subsets of Cohort 1 was 19 ( $S D=3.5)$. See Dawber [2] for a description of design of the Framingham Heart Study.

\section{Methods}

\section{Familial resemblance in Cohort I}

First we evaluated the distribution of AVGRES and found it to be significantly skewed and leptokurtotic in both males and females. A logarithmic (base 10) transformation rendered AVGRES normally distributed in both genders (for males, $p=0.35$, and for females, $p=0.46$ ). All subsequent analyses were carried out on the transformed values of AVGRES.

To assess family resemblance for AVGRES we used the FCOR program from the S.A.G.E. computer package [3]. Table 1 reports the gender-specific sib-pair correlations and their respective sample sizes. Modest, albeit significant, correlations in the range of 0.2 to 0.3 were obtained, and all are substantially higher than the spousal correlation of 0.045 .

\section{Linkage analysis}

Our plan was to analyze all available sib pairs from Cohort 1 for linkage with the objective of developing hypotheses that could be tested in Cohort 2. Unfortunately, only a small subset of the Cohort 1 subjects (that were used to assess family resemblance) were genotyped. The distribution of genotyped sibs is as follows: 38 pairs, 6 trios, and 1 quintet.

To perform multipoint quantitative linkage analysis for log AVGRES, we chose two methods implemented in the GENEHUNTER linkage program: nonparametric (NP) and expectation maximization Haseman-Elston (EM-HE). The NP method performs a Wilcoxon rank-sum test by first summing the ranks of absolute trait difference from sib pairs, multiplied by a simple weight based on the number of alleles shared identically by descent (IBD). Z scores are then obtained by the usual method [4].

Table I: Family resemblance for log AVGRES in cohort

\begin{tabular}{lrc}
\hline Relationship & N & Correlation \\
\hline Sister/Sister & 97 & 0.293 \\
Brother/Sister & 181 & 0.216 \\
Brother/Brother & 90 & 0.227 \\
Spouse & 348 & 0.045
\end{tabular}

The EM-HE procedure is based on the traditional Haseman-Elston method of regressing the squared sib-pair trait difference on the proportion of alleles shared IBD. In addition, when genotype information is missing, the EM algorithm infers the probability of alleles shared IBD by taking into account the allele sharing distribution as well as the regression parameters estimated from the real data points [5]. Similar to the NP score, the EM-HE test statistic for the regression coefficient is asymptotically normally distributed with mean 0 and unit variance. The GENEHUNTER 'pairs used' option was set to 3, which corresponds to 'all pairs of affected/phenotyped sibs.' This option weighs a sibship's contribution according to the number of independent pairs it contains, and guards against the possibility of large sibships dominating the results [6-9].

\section{Results \\ Cohort I}

Table 2 reports all markers that attained nominal significance at the $5 \%$ level (i.e., score $\geq 1.645$ ). Linkage signals at four markers were detected with just the NP statistic, 25 with just the EM-HE statistic; 11 signals were detected with both statistics.

\section{Cohort 2}

The members of Cohort 2 have a maximum of only five SBP assessments (taken over a period of about 20 years). We felt that performing the regression analysis on subjects with fewer than five measurements could not adequately capture the sort of variation we are interested in. Indeed, the relative paucity of Cohort 2 measures may render it fundamentally different from Cohort 1 . Restricting the analysis to genotyped sibs who were older than age 20 and had all five SBP assessments, however, yielded a much larger sample size compared to Cohort 1: 151 pairs, 84 trios, 35 quartets, 7 quintets, 4 sextets, and 2 septets.

Table 2 reports the NP and EM-HE linkage scores for those markers that attained nominal significance in Cohort 1 . As expected, few of the original signals could be validated. If we count as a "replication," markers with a $p$-value < 0.05 obtained under the same weighting and statistical methodology, then there are two replications: D5S1456 for the NP approach and D11S2359 for the EM-HE approach. Two additional markers (D1S1653 and D20S470) revealed a "reversal". Both of these markers gave a nominally significant signal in Cohort 1 with the EM-HE statistic, but in Cohort 2 it was the NP statistic that achieved nominal "replication".

\section{Extended pedigrees}

Combining available individuals from both cohorts results in 17 new families that were not analyzed in either cohort. Specifically, there are two half-sib pair families, 
Table 2: Significant NP and EM-Haseman-Elston scores from Cohort I and the "replication" scores from Cohort 2

\begin{tabular}{|c|c|c|c|c|c|}
\hline \multirow[b]{2}{*}{ Marker } & \multirow[b]{2}{*}{ Distance $^{A}$} & \multicolumn{2}{|c|}{ Cohort I } & \multicolumn{2}{|c|}{ Cohort 2} \\
\hline & & NP & EM-HE & NP & EM-HE \\
\hline DIS534 & 152 & 0.33 & 1.83 & 1.39 & 1.01 \\
\hline DISI653 & 164 & -0.04 & 1.87 & 1.98 & 1.47 \\
\hline D2S4I0 & 125 & 1.74 & 0.80 & -0.32 & -0.19 \\
\hline D2SI 384 & 200 & 1.17 & 2.00 & -0.89 & 0.44 \\
\hline D2S2944 & 210 & 1.12 & 1.88 & -0.96 & 0.70 \\
\hline D4S2632 & 51 & 1.19 & 1.80 & -0.16 & 0.20 \\
\hline D4S2394 & 130 & 1.71 & 0.57 & -0.26 & -0.35 \\
\hline D4SI 625 & 146 & 1.71 & 0.46 & 0.13 & -0.16 \\
\hline D5SI456 & 175 & 1.90 & 1.18 & 2.45 & 2.40 \\
\hline D9SIIII & 44 & 1.00 & 1.83 & -0.14 & 1.16 \\
\hline GATA I 2 IA08 & 88 & 1.44 & 2.12 & -0.44 & -0.26 \\
\hline DIOSI432 & 94 & 1.42 & 2.46 & -0.45 & 0.15 \\
\hline DIOS2327 & 101 & 1.36 & 2.14 & 0.64 & 1.00 \\
\hline DIOS2470 & 113 & 0.33 & $\mathrm{I} .74$ & 0.74 & -0.41 \\
\hline DIOSI230 & 143 & 1.41 & 1.68 & 2.36 & 2.51 \\
\hline DIIS2359 & 147 & 1.78 & 2.12 & 1.36 & 3.29 \\
\hline DI4S742 & 12 & 1.67 & 3.00 & 0.22 & -1.83 \\
\hline DI4SI 280 & 26 & 0.93 & 1.79 & -0.03 & -0.65 \\
\hline DI4S608 & 28 & 0.98 & 1.89 & 0.00 & -0.26 \\
\hline DI4S297 & 32 & 0.86 & 2.14 & 0.03 & -0.47 \\
\hline DI4S53(A) & 85 & 0.92 & 1.86 & -0.55 & -0.69 \\
\hline DI4S606 & 92 & 1.65 & 2.75 & -0.51 & -0.09 \\
\hline GATAI93A07 & 96 & 2.57 & 3.29 & -0.80 & -0.07 \\
\hline DI4S6I0 & 97 & 2.58 & 3.30 & -0.90 & -0.13 \\
\hline DI4S6I7 & 106 & 1.40 & 1.73 & -1.73 & -0.58 \\
\hline DI4SI434 & 113 & 1.64 & 2.34 & -1.31 & -0.34 \\
\hline DI6S2624 & 88 & 1.05 & 2.16 & 0.30 & 0.62 \\
\hline GATAI78FII & 3 & 2.89 & 4.19 & 0.63 & 0.69 \\
\hline DI8S48I & 7 & 3.00 & 4.53 & -0.05 & 0.01 \\
\hline DI8S976 & 13 & 3.03 & 3.74 & -0.71 & -0.54 \\
\hline DI8S843 & 28 & 2.36 & 3.16 & -1.51 & -1.70 \\
\hline DI8S542 & 41 & 2.27 & 2.20 & -1.07 & -1.75 \\
\hline DI8S877 & 54 & 2.02 & 3.21 & -0.90 & -1.47 \\
\hline DI8S535 & 64 & 1.89 & 2.97 & -0.82 & -0.98 \\
\hline DI9SI034 & 21 & 1.45 & 1.81 & 0.16 & 0.40 \\
\hline DI9S589 & 88 & 1.54 & 2.26 & -0.41 & 1.31 \\
\hline DI9S254 & 101 & 1.18 & 2.06 & -0.63 & 1.11 \\
\hline D20S470 & 39 & 0.82 & 2.22 & 2.23 & 1.57 \\
\hline D20S477 & 48 & 0.60 & 1.97 & 1.50 & 1.64 \\
\hline D20S478 & 54 & 0.80 & 1.86 & 1.10 & 1.42 \\
\hline
\end{tabular}

ADistance is from p-arm telomere of the sex-average Marshfield map http://research.marshfieldclinic.org/genetics/. Duplicate marker D I4S53 was retained for analysis but deleted from this table.

two sib-pair families (that straddle the cohorts), and 13 families with additional avuncular or cousin members. Five large pedigrees were disassembled at a marriage node into smaller units. A total of 305 families were used for the combined analyses.

Table 3 reports the results of the multipoint linkage analysis in the extended pedigrees for the four "replicated" markers (and their closest neighbors). Compared to the
$\mathrm{NP}$ and EM-HE scores obtained from the sib-pair analysis of Cohort 2, the scores for the extended families reflect some degradation, although all but D1S1653 remain nominally significant by one or the other method.

\section{Discussion}

Physiological homeostasis is the process whereby a narrow range of phenotypes develops in the presence of wide variation in genotypes and environments. A classic exam- 
Table 3: Linkage analysis of extended families for markers with significant evidence in Cohorts I and $\mathbf{2}^{\mathrm{A}}$

\begin{tabular}{|c|c|c|c|}
\hline Marker & Distance & NP & EM-HE \\
\hline DIS534 & 152 & 0.89 & 0.81 \\
\hline DISI653 & 164 & 1.62 & 1.18 \\
\hline DISI679 & 171 & 1.60 & 1.40 \\
\hline D5SI47I & 172 & 1.54 & 1.92 \\
\hline D5SI456 & 175 & 2.05 & 2.14 \\
\hline D5S2II & 183 & 2.54 & 1.86 \\
\hline DIIS9I2 & $|3|$ & 1.20 & 1.47 \\
\hline DIIS2359 & 147 & $\mathrm{I} .47$ & 2.86 \\
\hline D20S604 & 33 & 0.78 & 0.60 \\
\hline D20S470 & 39 & 1.85 & 1.08 \\
\hline D20S477 & 48 & 1.91 & 1.60 \\
\hline
\end{tabular}

AMultipoint scores for the closest proximal and distal markers (except for DIIS2359, which has no telomeric neighbor) are included.

ple is the ability of homeothermic mammals to maintain a constant body temperature despite substantial genetic variation and fluctuating ambient air temperatures. The concept of physiological homeostasis bears some similarity to Waddington's notion of "canalization" [10] although this latter concept is usually applied to growth and developmental homeostasis.

That a breakdown in physiological homeostasis can result in either an increase or decrease in a phenotype - thus increasing the variance - is recognized in the operational definitions of many disorders. For example, among the Diagnostic and Statistical Manual of Mental Disorders (4th ed.) criteria for major depression are the following: a significant decrease or increase in appetite, insomnia or hypersomnia, psychomotor agitation or psychomotor retardation.

While the preponderance of genetic studies concentrate on the first moment of various phenotypes - and for medical reasons are usually interested in understanding deviantly high (or, rarely, deviantly low) phenotypes - it is reasonable to suppose that the magnitude of an individual's phenotypic variation is to some extent under genetic control [11]. Indeed, recently it has been shown that with respect to short-term (24-hour) variation among hypertensive patients, variability in blood pressure is positively related to organ damage and cardiovascular morbidity [12].

We used the rich longitudinal Framingham Heart Study data to study the genetics of long term variation in SBP. Our findings indicate that there is moderate familial resemblance for the magnitude of the deviation of a person's SBP from his or her unique age-related trend line. At least three caveats need to be mentioned. First, we restricted our Cohort 1 analysis to individuals with 10 or more measurements. Because this cohort was relatively small, we chose to include all subjects who met the above criterion, regardless of whether they were under treatment for hypertension. Subsequent to the Genetic Analysis Workshop 13, however, we carried out an analysis to determine if there exists a relationship between medication usage and AVGRES. We quantified medication usage as the proportion of SBP assessments where the subject was reported to be medicated. Unexpectedly, there is a strong positive correlation for both cohort $1(r=0.51)$ and Cohort $2(r=0.29)$ in the Framingham Heart Study. This previously unrecognized relationship between the use of hypertension medication and AVGRES is likely to have confounded our linkage analysis in an unknown fashion. Second, we did not adjust the data for body mass index (BMI)-a covariate known to show familial resemblance and to affect SBP. Since our phenotype was defined as the average residual from each subject's unique regression line it is unclear what, if any, effect the failure to adjust for BMI had on our results. Third, we did not test whether curvilinear regression would have provided an improved fit compared to simple linear regression. This decision was based on two considerations. First, if for some of the subjects, a quadratic or cubic function were found to fit significantly better than the linear component, its use in a subset of the data would have created a heterogeneous definition of the phenotype. Second, we were aware that the maximum number of available SBP measures on the Cohort 2 subjects was five, and it seemed frivolous to fit a high order function to such meager data.

For the linkage analysis, we chose to analyze the sibship data with two different methods. Although the NP and the EM-HE methods are similar, as are all linkage methods, they are not identical. For the data we analyzed, the correlation between the NP and EM-HE scores were 0.72 and 


\section{Cohort 1}

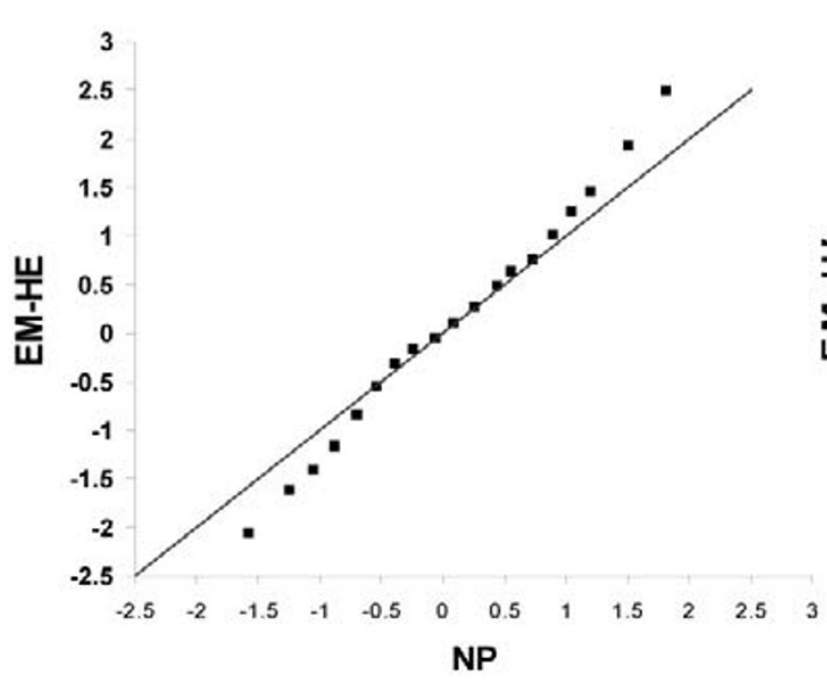

Cohort 2

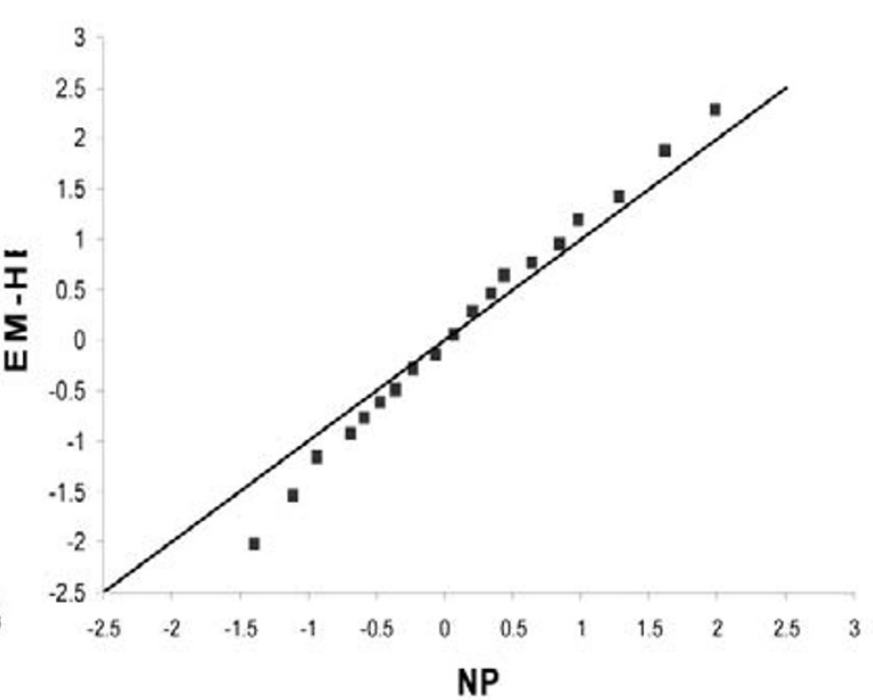

Figure I

Quantile-quantile plots in 5\% increments of the linkage statistics for Cohort I and Cohort 2

0.69 for Cohorts 1 and 2, respectively. Inspection of the quantile-quantile (QQ) plots (Fig. 1) reveals that the NP statistic is more conservative than the EM-HE statistic. Indeed, $11.6 \%$ and $11.3 \%$ of the markers in Cohorts 1 and 2, respectively, are significant at the 0.05 level with EM-HE, whereas $6.0 \%$ and $7.5 \%$ are significant with the NP statistic.

We used the linkage analysis on Cohort 1 to develop hypothesis that could be tested in Cohort 2. Two genomic regions were "replicated" in Cohort 2 using the same statistical method (D5S1456 and D11S2359). An additional two signals were found to be nominally significant by EMHE in Cohort 1 and "replicated" with NP in Cohort 2. Three of these four signals remained nominally significant when extended pedigrees were analyzed.

\section{Acknowledgments}

This work was supported in part by the Urologic Research Foundation. Some of the results reported here were obtained by using the program package S.A.G.E., which is supported by U.S. Public Health Resource Grant RR 03655 from the National Center for Research Resources.

\section{References}

I. Levy D, DeStefano AL, Larson MG, O'Donnell C], Lifton RP, Gavras $H$, Cupples LA, Myers RH: Evidence for a gene influencing blood pressure on chromosome 17: genome scan linkage results for longitudinal blood pressure phenotypes in subjects from the Framingham Heart Study. Hypertension 2000, 36:477-483.
2. Dawber TR: The Framingham Study: The Epidemiology of Atherosclerotic Disease. Cambridge, MA, Harvard University Press 1980.

3. S.A.G.E.: Statistical Analysis for Genetic Epidemiology, Release 3.I Department of Epidemiology and Biostatistics, MetroHealth Campus, Case Western Reserve University, Cleveland, $\mathrm{OH} ; 1997$.

4. Kruglyak L, Lander ES: A nonparametric approach for mapping quantitative trait loci. Genetics 1995, I39: I42 I- I 428.

5. Kruglyak L, Lander ES: Complete multipoint sib-pair analysis of qualitative and quantitative traits. Am J Hum Genet 1995, 57:439-454.

6. Suarez BK, Hodge SE: A simple method to detect linkage for rare recessive diseases: an application to juvenile diabetes. Clin Genet 1979, I 5: 126-136.

7. Fernandez JR, Etzel C, Beasley TM, Shete S, Amos Cl, Allison DB: Improving the power of sib pair quantitative trait loci detection by phenotype winsorization. Hum Hered 2002, 53:59-67.

8. Davis S, Weeks DE: Comparison of nonparametric statistics for detection of linkage in nuclear families: single-marker evaluation. Am J Hum Genet 1997, 61: |431-1444.

9. Hodge $\mathrm{S}$ : The information contained in multiple sibling pairs. Genet Epidemiol 1984, I:109-122.

10. Waddington $\mathrm{CH}$ : The Strategy of the Genes. London, Allen and Unwin 1957.

II. Coady SA, Jaquish CE, Fabsitz RR, Larson MG, Cupples LA, Myers RH: Genetic variability of adult body mass index: a longitudinal assessment in Framingham families. Obesity Res 2002, 10:687-68I.

12. Sega R, Corrao G, Bombelli M, Beltrame L, Facchetti R, Grassi G, Ferrario $M$, Mancia $G$ : Blood pressure variability and organ damage in a general population. Results from the PAMELA study. Hypertension 2002, 39:710-7|4. 